\title{
Pisacano Leadership Foundation
}

The American Board of Family Medicine (ABFM) established the Pisacano Leadership Foundation (formerly the Nicholas J. Pisacano, MD, Memorial Foundation) in 1991 in tribute to the founder and first Executive Director of the ABFM. The Pisacano Scholars Leadership Program provides leadership training and scholarship funding for outstanding fourth-year medical students committed to the specialty of Family Medicine. The Foundation gives special recognition to the following donors (the list includes all contributions received July 1, 2008, to July 1, 2009):

\section{$\$ 10,000+$}

Anonymous

\section{\$1000-\$9999}

Gaynell Anderson, MD

BB\&T Charitable Foundation

Erika B. Bliss, MD

Cheng-Chieh Chuang, MD

Eric Crall, MD

Betsy A. Garrett, MD

Wanda C. Gonsalves, MD

Deborah G. Haynes, MD

William J. Kane, MD

Kurt A. Lindberg, MD

James C. Martin, MD

Karen B. Mitchell, MD

Trish Palmer, MD

James C. Puffer, MD

\section{\$500-\$999}

William G. Bernhardt, MD

Elizabeth A. Burns, MD

Stephen G. Cook, MD

Sabina Diehr, MD

Linda French, MD

Kenneth Grimm, MD

Michael D. Hagen, MD

Keith Knepp, MD

Jennifer E. Lochner, MD

Corey Martin, MD
Michael D. Needleman, MD

Wilner N. J. Nelson, MD

Thomas O'Neill, PhD

Janet P. Realini, MD

Jill E. Tomek, MD

James D. Toombs, MD

Barbara E. Troupin, MD

\section{\$250-\$499}

Suzanne M. Allen, MD

Louise Berner-Holmberg, MD

Katrina P. Bradford, MD

Alvah R. Cass, MD

Max A. Crocker, MD

Brian H. Gair, MD

Gary A. Goforth, DO

John R. Hayes, MD

Jim Henry

Jerome J. Hotchkiss, Jr., MD

Samuel M. Jones, MD

Nerissa N. Koehn, MD

Patricia Lebensohn, MD

Gary M. Plant, MD

Susan L. Powell, MD

Howard K. Rabinowitz, MD

Jamie L. Reedy, MD

Seth P. Rosebrock

W. Jack Stelmach, MD

Marti Y. Taba, MD

David Turner, MD 
George C. Xakellis, Jr., MD

Edward M. Yu, MD

\section{\$100-\$249}

Vincent P. Ang, MD

William L. Babaian, MD

Joan M. Bedinghaus, MD

Timothy M. Beittel, MD

Robert P. Blereau, MD

James F. Calvert, MD

Lucy M. Candib, MD

E. G. Cline, MD

Craig Czarsty, MD

Charles J. de Sieyes, MD

Marguerite Duane, MD

Joshua Freeman, MD

Sim Galazka, MD

Valerie J. Gilchrist, MD

Ronald H. Goldschmidt, MD

Victoria A. Gorski, MD

Larry A. Green, MD

David M. Harsha, MD

James P. Jarboe, MD

Rick D. Kellerman, MD

Richard D. Kiovsky, MD

Scott D. Kirsch, MD

Shou Ling Leong, MD

Patrick Y. Leung, MD

Sean Lucan, MD

Richard F. Madden, MD

Michael K. Magill, MD

Kenneth E. Mayeda, MD

Darlyne Menscer, MD

Katherine M. Miller, MD

Ronald A. Miller, MD

Peter M. Nalin, MD

Nancy E. Morden, MD

Thomas E. Norris, MD

John Pakula, MD

John J. Pastore, MD

Lisa M. Peterson, MD
Michele P. Pugnaire, MD

Sumathy Reddy, MD

David A. Rinehart, MD

Thomas L. W. Roe, MD

Saria C. Saccocio, MD

Dennis F. Saver, MD

Ellsworth C. Seeley, MD

William B. Shore, MD

J. Lewis Sigmon, Jr., MD

Samuel K. Suttle, MD

Van E. Vahle, MD

James P. Valek, MD

Gerald L. Van Es, MD

Gregg A. Warshaw, MD

Raymond K. Weber, MD

Sandford L. Weiler, MD

Allan J. Wilke, MD

Peter C. $\mathrm{Wu}, \mathrm{MD}$

Nikitas J. Zervanos, MD

\section{Up to $\$ 99$}

Victor Lee Allen, MD

Kenneth N. Andersen, MD

Robert A. Baldor, MD

Nahum M. Balotin, MD

Frank A. Beardsley, MD

Suzanne Cashman, MD

Jason Chao, MD

Harry Collins, MD

James P. Crews, MD

Jennifer E. DeVoe, MD

Allen J. Dietrich, MD

Adelmo P. Dunghe, MD

Krista Farey, MD

Constantine E. Flokas, MD

David R. Garr, MD

Lillian Gelberg, MD, MSPH

John P. Geyman, MD

Amy M. Givler, MD

Patricia T. Glowa, MD

James E. Gottemoller, MD 
Laura Gottlieb, MD

A. Meryl Grasse, MD

Tracy Kedian, MD

John E. Kelly, MD

John P. Kowalski, MD

Alma B. Littles, MD

A. Gabriel Maijub, MD, PhD

Harold Marshall

Joel H. Merenstein, MD

Margaret L. Moore, MD

Sarah E. Morgan, MD

Elizabeth Naumburg, MD

Duane L. Orn, MD

Barrie Paster, MD
Theodore J. Phillips, MD

William R. Phillips, MD

Samuel C. Pickens, MD

H. Dusty Rhodes, DO

Alan S. Rogers, MD

Gilbert A. Rude, MD

Rebecca Steckel, MD

Keith L. Stelter, MD

John F. Tabachnick, MD

Joseph F. Termini, MD

Robert E. Tortolani, MD

Daniel A. Walters, MD

Shiush C. Wang, MD

Susan Wickes, MD, and Robert Torassa, Jr.

Jane Ireland

Pisacano Leadership Foundation 